\title{
Preparo de antibióticos em uma unidade de neonatologia: Análise sobre os cuidados de enfermagem
}

\author{
Preparation of antibiotics in a Neonatology: Analysis on nursing care \\ Preparación de antibióticos en um Neonatología: Análisis de la atención de enfermería
}

Recebido: 14/04/2021 | Revisado: 19/04/2021 | Aceito: 23/04/2021 | Publicado: 09/05/2021

Leticia Alexandre Lima

ORCID: https://orcid.org/0000-0003-2566-0315 Universidade Estadual do Ceará, Brasil

E-mail: leticia_lima1@hotmail.com

Lia Bezerra Furtado Barros

ORCID: https://orcid.org/0000-0002-6256-2901 Universidade Estadual do Ceará, Brasil E-mail: lia.bfb@gmail.com

Patrícia do Nascimento César Bessa ORCID: https://orcid.org/0000-0002-3145-7930

Universidade Estadual do Ceará, Brasil

E-mail: patricianascimentoc@gmail.com

Débora Pena Batista e Silva

ORCID: https://orcid.org/0000-0003-0633-5242 Universidade Estadual do Ceará, Brasil

E-mail: pbs.debora@gmail.com

Débora Silveira de Lima

ORCID: https://orcid.org/0000-0002-4841-2333 Universidade Estadual do Ceará, Brasil

E-mail: debora.s1001@gmail.com

Ana Caroline Araujo da Silva

ORCID: https://orcid.org/0000-0001-7864-8761

Universidade Estadual do Ceará, Brasil

E-mail: anacaroline222324@gmail.com

Samila Sousa Vasconcelos

ORCID: https://orcid.org/0000-0002-1509-1887

Centro Universitário Inta - Uninta, Brasil

E-mail: samilasousa@ @otmail.com

Cláudia Patrícia da Silva Ribeiro Menezes

ORCID: https://orcid.org/0000-0002-0559-9302 Universidade Estadual do Ceará, Brasil E-mail: claudia_ribeiro6@hotmail.com

Lívia Lopes Custodio

ORCID: https://orcid.org/0000-0001-9610-7379 Universidade Estadual do Ceará, Brasil E-mail: liviacustodio@yahoo.com.br

Débora Cristina Couto Oliveira Costa

ORCID: https://orcid.org/0000-0002-9402-0613 Universidade Estadual do Ceará, Brasil

E-mail: deboraccoc@gmail.com

Ilvana Lima Verde Gomes

ORCID: https://orcid.org/0000-0002-1861-5121 Universidade Estadual do Ceará, Brasil

E-mail: ilverde@gmail.com

\section{Resumo}

As boas práticas para o uso seguro de medicamentos são direcionadas para a prevenção do erro de medicação no cuidado prestado ao recém-nascido por se tratar de uma população frágil e vulnerável. Objetivou-se analisar a existência de conformidades e não conformidades no preparo de antibiótico por enfermeiros em uma unidade de neonatologia. A pesquisa é do tipo transversal e descritiva de natureza observacional, desenvolvida com 12 enfermeiras de um hospital terciário. A coleta de dados aconteceu em janeiro de 2020 por meio de observação do preparo de medicação e dois formulários. O primeiro instrumento abordou dados do ambiente, do procedimento, rotulação correta e preparado do medicamento; o segundo coletou informações socioprofissionais. Os dados foram organizados no Excel e analisados pela estatística descritiva, com números inteiros, porcentagens, medidas da média e desvio padrão. Os resultados demonstraram que as enfermeiras apresentaram média de idade 37,83 anos; com 12,3 
anos de formação acadêmica; 58,3\% possuindo pós-graduação; 8,7 anos de experiência profissional, a maioria cooperados $(75 \%)$. Quanto aos conhecimentos e práticas da equipe, viu-se a não utilização de prescrição, nem uso do rótulo de medicação; manipulação de forma incorreta; preparo com antecedência, mas houve conformidades com relação ao ambiente, a iluminação e ao uso de EPIs. Conclui-se que as não conformidades se sobressaem e nos levam a refletir sobre a atuação profissional do enfermeiro com vínculo empregatício frágil e no poder da gestão de interferir positivamente na promoção de um ambiente seguro, desencadeado por uma cultura de segurança institucionalizada.

Palavras-chave: Cuidados de enfermagem; Antibacterianos; Erros de medicação; Neonatologia.

\begin{abstract}
Good practices for the safe use of medications are aimed at preventing medication errors in the care provided to the newborn, as it is a fragile and vulnerable population. The objective was to analyze the existence of conformities and non-conformities in the preparation of antibiotics by nurses in a neonatology unit. The research is cross-sectional and descriptive of an observational nature, developed with 12 nurses from a tertiary hospital. Data collection took place in January 2020 through observation of medication preparation and two forms. The first instrument addressed data on the environment, the procedure, correct labeling and preparation of the medication; the second collected socioprofessional information. The data were organized in Excel and analyzed using descriptive statistics, with integers, percentages, measures of the mean and standard deviation. The results showed that the nurses had an average age of 37.83 years; with 12.3 years of academic training; $58.3 \%$ have graduate degrees; 8.7 years of professional experience, most of them cooperative $(75 \%)$. As for the team's knowledge and practices, there was no use of a prescription, nor use of the medication label; incorrect handling; preparation in advance, but there were conformities regarding the environment, lighting and the use of PPE. It is concluded that non-conformities stand out and lead us to reflect on the professional performance of nurses with fragile employment and the power of management to positively interfere in the promotion of a safe environment, triggered by an institutionalized safety culture.
\end{abstract}

Keywords: Nursing care; Anti-Bacterial agents; Medication error; Neonatology.

\title{
Resumen
}

Las buenas prácticas para el uso seguro de medicamentos están orientadas a prevenir errores de medicación en la atención brindada al recién nacido, por tratarse de una población frágil y vulnerable. El objetivo fue analizar la existencia de conformidades y no conformidades en la preparación de antibióticos por enfermeras en una unidad de neonatología. La investigación es transversal y descriptiva de carácter observacional, desarrollada con 12 enfermeras de un hospital terciario. La recolección de datos se llevó a cabo en enero de 2020 mediante la observación de la preparación de medicamentos y dos formas. El primer instrumento abordó datos sobre el medio ambiente, el procedimiento, el correcto etiquetado y preparación de la medicación; el segundo recopilaba información socioprofesional. Los datos se organizaron en Excel y se analizaron mediante estadística descriptiva, con números enteros, porcentajes, medidas de la media y desviación estándar. Los resultados mostraron que las enfermeras tenían una edad promedio de 37,83 años; con 12,3 años de formación académica; El 58,3\% tiene títulos de posgrado; 8,7 años de experiencia profesional, la mayoría cooperativa (75\%). En cuanto a los conocimientos y prácticas del equipo, no se utilizó prescripción ni etiqueta del medicamento; manejo incorrecto; preparación con anticipación, pero hubo conformidades con respecto al medio ambiente, iluminación y uso de EPI. Se concluye que destacan las no conformidades y nos llevan a reflexionar sobre el desempeño profesional del enfermero con empleo frágil y el poder de la gestión para interferir positivamente en la promoción de un ambiente seguro, desencadenado por una cultura de seguridad institucionalizada.

Palabras clave: Atención de enfermería; Antibacterianos; Errores de medicación; Neonatología.

\section{Introdução}

Estima-se que pacientes neonatais sejam, pelo menos, três vezes mais propensos a sofrer danos causados por erros de medicação do que os adultos. A segurança do paciente encontra-se no cuidado seguro. Segundo a Organização Pan-Americana de Saúde (OPAS) e a Organização Mundial de Saúde (OMS), os erros de medicação geram custos de 42 milhões de dólares por ano e causam, pelo menos, uma morte por dia, considerando-se, assim, um problema de saúde pública mundial. Diante deste cenário e com o intuito de reduzir em 50\% danos graves e evitáveis até o ano de 2022, a OMS, trouxe a Medicação de Segurança como tema do terceiro Desafio Global da Segurança do Paciente (WHO, 2017).

O erro de medicação é descrito como "qualquer evento evitável que pode causar ou levar ao uso inadequado de medicamentos ou danos ao paciente enquanto o medicamento está sob o controle do profissional de saúde", de acordo com National Coordinating Council for Medication Error Reporting and Prevention (2015). Caso haja aumento dos erros, elevam-se os custos com a internação hospitalar de longa permanência e causam impacto sobre a morbimortalidade dos pacientes 
(Popescu et al., 2011).

Dentre as classes de medicamentos, os antibióticos apresentam graves consequências quando estão envolvidos em erros de medicações. Nas últimas duas décadas, houve um aumento na prevalência de infecções bacterianas multidrogarresistentes que estão relacionadas ao cuidado em saúde. Esse fato tornou o uso racional de antimicrobianos uma das estratégias da OMS para prevenir o surgimento de resistência a antibióticos, ao mesmo tempo em que melhora os desfechos e a segurança do paciente (Embree \& Alfattoh, 2018).

Nesse contexto, a segurança do paciente atrelada a saúde do trabalhador promove a assistência em saúde de qualidade. O erro no cuidado em saúde é desencadeado por um conjunto de aspectos que envolvem toda a rede de atendimento ao paciente, desde o ato humano até condições de trabalho adequadas.

A instituição de saúde deve prover um ambiente reservado para leitura e preparo de medicamentos, em boas condições de higiene, iluminado, com circulação de ar e principalmente, baixo nível de ruído. Deve ainda, estar longe de qualquer fonte de distração, com mobiliário e estrutura que facilitem a execução das tarefas e a postura corporal pela equipe de enfermagem (Feldman et al., 2017).

As não conformidades associadas ao manejo de antimicrobianos afetam a segurança e eficácia do tratamento devido ao emprego de esquemas posológicos equivocados, bem como a aplicação da semiotécnica inadequada no preparo de antimicrobianos, como, por exemplo, a não desinfecção dos frascos, dispersão de antibacterianos no ar, entre outras causas (Pereira et al., 2016).

No âmbito da neonatologia, a habilidade técnica e experiência no cuidado prestado ao recém-nascido (RN), mostramse ainda mais imprescindíveis, visto que, por se tratar de uma população frágil e vulnerável, qualquer manejo inadequado na assistência pode causar prejuízo com danos irreparáveis, sendo necessária a revisão criteriosa do saber-fazer do profissional de enfermagem (Guzzo et al., 2018).

Diante do exposto, o papel do enfermeiro atuante nas unidades de neonatologia (UN) é de grande responsabilidade na interceptação de erros que, mesmo antes da administração da medicação, podem ocasionar danos ao cliente, prejudicando a recuperação da saúde. Assim, as boas práticas para o uso seguro de medicamentos são direcionadas para a prevenção do erro de medicação através da cautela no processo assistencial desenvolvido pelo enfermeiro especialista em neonatologia (Guzzo et al., 2018).

Desse modo, este estudo é norteado pela pergunta-problema: Como ocorre o processo de preparo de antimicrobianos por enfermeiros em unidade de neonatologia? Quais são as conformidades e não conformidades encontradas em unidade de neonatologia, relacionadas ao preparo de antibióticos realizados por enfermeiros? Traçou-se assim, o objetivo desse estudo que é avaliar o preparo de antimicrobianos por enfermeiros em unidade de neonatologia.

\section{Metodologia}

Estudo do tipo quantitativo, transversal e descritivo de natureza observacional. A observação foi sistemática, na qual o pesquisador utiliza-se de um instrumento para guiá-lo na observação não participante. $\mathrm{O}$ estudo foi desenvolvido em um hospital de ensino terciário, de alta complexidade, com UN composta de: Unidade de Terapia Intensiva Neonatal (UTIN) e Unidade de Cuidado Intermediário Neonatal Convencional (UCINCo).

O hospital possui duas UTIN, cada uma com 10 leitos e duas UCINCo, a UCINCo I com 14 leitos e a UCINCo II com 22 leitos. Totalizando a contagem de leitos da UN I com 24 leitos e da UN II com 32 leitos cadastrados no Cadastro Nacional de Estabelecimentos de Saúde (CNES). Diante deste cenário, a pesquisa foi realizada na UCINCo II por conter o maior número de leitos, consequentemente, um maior quantitativo de preparo de antibióticos.

A população da pesquisa, inicialmente, foi composta por 15 enfermeiras plantonistas que são responsáveis pela 
diluição dos medicamentos no início de cada plantão e que trabalham na UCINCo II. A amostra foi selecionada por amostragem não probabilística quando a seleção de unidades amostrais não é aleatória, determinada por ordem do pesquisador, coletada na semana, carga horária do programa de residência em neonatologia, em plantões noturnos e diurnos. Por troca de plantões e licenças, resultou em uma amostra de 12 enfermeiras.

A coleta de dados foi realizada no mês de janeiro de 2020, tendo como critérios de inclusão: ser profissional da enfermagem; ter pelo menos dois anos de experiência na área de neonatologia; trabalhar na unidade, lócus desta pesquisa; ser plantonista; trabalhar durante a semana e que aceitassem participar da pesquisa voluntariamente. Como critério de exclusão, foram considerados: profissionais que se encontravam de férias, em licença médica ou licença maternidade, os enfermeiros residentes e os profissionais recém-admitidos.

Os dados foram coletados no início de cada plantão diurno e noturno de 12 horas, onde os (as) enfermeiros (as) plantonistas realizam a diluição dos medicamentos necessários para o período. São realizados, em 24 horas, duas diluições em quantidade suficiente para suprir a necessidade dos RN da UCINCo II e da UTIN II.

A primeira diluição é realizada no frasco de antibiótico e a segunda diluição é realizada e mantida na seringa por 12h sob-refrigeração. Todas as medicações que poderão ser utilizadas na unidade de neonatologia encontram-se reunidas em uma pasta, onde nas folhas contêm os itens: princípio ativo/apresentação; informações sobre o medicamento; soluções compatíveis para diluição; volume/reconstituição; estabilidade após a diluição; informações sobre incompatibilidade e pH.

Os instrumentos de coleta de dados foram dois formulários. O primeiro é um instrumento de observação do preparo de medicação, validado por um grupo de pesquisadores do projeto temático e multicêntrico de Cassiani et al. (2006) que foi adaptado para esta pesquisa com base na literatura pertinente ao assunto. Abordando em dados do ambiente: iluminação, nível de ruído, interrupções, espaço, local para higiene das mãos, limpeza de superfícies/bancadas de preparo, organização; em dados do procedimento temos a técnica correta (semiotécnica) que inclui lavagem das mãos, desinfecção de frasco-ampolas, reutilização de matérias (seringas e agulhas), contaminação de material e/ou medicamento, preparo com muita antecedência e proteção de medicamentos fotossensíveis; por fim, rotulação correta do medicamento preparado.

O segundo formulário coletou informações socioprofissionais dos enfermeiros que participaram da pesquisa com base no mesmo estudo citado anteriormente, acrescido de algumas questões pertinentes, levantadas pelas pesquisadoras e específicas para a população.

Foram aplicados 12 questionários e 12 observações de diluição. Antes de cada diluição de antibióticos foi realizado a aplicação do formulário para coletar os dados socioprofissionais através de questionário estruturada. Já para a aplicação do formulário de preparo de medicamento, foi utilizado a observação do tipo participante, na qual a pesquisadora, em caso de erro no preparo do antibiótico, interrompia o procedimento a fim de preservar a segurança do paciente.

Os dados foram organizados e analisados no software Microsoft Excel 2016, através de estatística descritiva, com números inteiros, porcentagens, medidas da média e desvio padrão. O projeto da pesquisa obedeceu às normas referidas pela Resolução 466/12 do Conselho Nacional de Saúde CNS que fórmula as diretrizes para pesquisas que envolvem seres humanos. Os profissionais que participaram do estudo assinaram o Termo de Consentimento Livre e Esclarecido (TCLE). Tem como Certificado de Apresentação e Apreciação Ética o número: 26234619.4.0000.5041.

\section{Resultados}

A aplicação do formulário foi realizada com 12 enfermeiras, com média de idade 37,83 anos e todas do sexo feminino. A média de tempo de formação acadêmica foi de 12,3 anos, com 58,3\% dos profissionais possuindo título de pósgraduação (especialista, mestre e/ou doutor). No âmbito da neonatologia, o tempo de experiência profissional foi, em média, de 8,7 anos e maior vínculo empregatício no hospital foi de profissionais cooperados (75\%). 
Tabela 1 - Perfil socioprofissional dos enfermeiros coletado por formulário estruturado - Fortaleza, Ceará, Brasil - 2020.

\begin{tabular}{|c|c|c|c|c|}
\hline Características & $\mathbf{N}$ & $\%$ & Média & Desvio padrão \\
\hline \multicolumn{5}{|l|}{ Idade (anos) } \\
\hline $20-30$ & 2 & 16,7 & \multirow{5}{*}{37,83} & \\
\hline $31-40$ & 7 & 58,3 & & \\
\hline $41-50$ & 2 & 16,7 & & 10,53 \\
\hline $51-60$ & 0 & 0,0 & & \\
\hline $61-70$ & 1 & 8,3 & & \\
\hline \multicolumn{5}{|l|}{ Gênero } \\
\hline Feminino & 12 & 100,0 & & \\
\hline Masculino & 0 & 0,0 & & \\
\hline \multicolumn{5}{|l|}{ Formação acadêmica } \\
\hline Ensino Superior Completo & 5 & 41,7 & & \\
\hline Pós-graduado & 5 & 41,7 & & \\
\hline Mestrado & 1 & 8,3 & & \\
\hline Doutorado & 1 & 8,3 & & \\
\hline Pós-Doutorado & 0 & 0,0 & & \\
\hline \multicolumn{5}{|l|}{ Tempo de formação acadêmica (anos) } \\
\hline 01 a 05 & 2 & 16,7 & \multirow[t]{5}{*}{12,3} & \\
\hline 06 a 10 & 7 & 58,3 & & \\
\hline 11 a 15 & 0 & 0,0 & & \\
\hline 16 a 20 & 2 & 16,7 & & \\
\hline$>20$ & 1 & 8,3 & & \\
\hline \multicolumn{5}{|l|}{ Tempo de experiência profissional(anos) } \\
\hline 01 a 05 & 4 & 33,3 & \multirow{5}{*}{8,7} & \multirow{5}{*}{10,31} \\
\hline 06 a 10 & 7 & 58,3 & & \\
\hline 11 a 15 & 0 & 0,0 & & \\
\hline 16 a 20 & 0 & 0,0 & & \\
\hline$>20$ & 1 & 8,3 & & \\
\hline \multicolumn{5}{|l|}{ Vínculo institucional } \\
\hline Público & 3 & 25,0 & \multirow{2}{*}{7,16} & \\
\hline Contratado/cooperado & 9 & 75,0 & & \\
\hline
\end{tabular}

Fonte: Autores (2020).

Quanto à observação do ambiente utilizado para diluição de medicamentos, foi caracterizado como adequado e inadequado. Observou-se que ruído, espaço, organização do ambiente e limpeza da superfície de preparo foram, predominantemente, inadequados. Apenas a iluminação é tida como adequada (100\%) para a execução do preparo de antibióticos.

Os dados da Tabela 2 apontam que todas as enfermeiras foram interrompidas durante o preparo de antibióticos e quais foram às causas de interrupções, revelando que as interrupções por outro profissional foi a maior causa (93,5\%) com um total de 123 interrupções. 
Tabela 2 - Distribuição percentual, conforme os dados analisados no ambiente e os tipos de interrupções durante o preparo de antibióticos - Fortaleza, Ceará, Brasil - 2020.

\begin{tabular}{|c|c|c|}
\hline Dados do ambiente & $\mathbf{N}$ & $\%$ \\
\hline \multicolumn{3}{|l|}{ Iluminação } \\
\hline Adequada & 2 & 100,0 \\
\hline Inadequada & 0 & 0,0 \\
\hline \multicolumn{3}{|l|}{ Ruído } \\
\hline Adequado & 0 & 0,0 \\
\hline Inadequado & 12 & 100,0 \\
\hline \multicolumn{3}{|l|}{ Espaço } \\
\hline Adequado & 1 & 8,3 \\
\hline Inadequado & 11 & 91,7 \\
\hline \multicolumn{3}{|l|}{ Organização do ambiente } \\
\hline Adequada & 5 & 41,7 \\
\hline Inadequada & 7 & 58,3 \\
\hline \multicolumn{3}{|l|}{ Limpeza da superfície de preparo } \\
\hline Adequada & 5 & 41,7 \\
\hline Inadequada & 7 & 58,3 \\
\hline \multicolumn{3}{|l|}{ Interrupções } \\
\hline Sim & 12 & 100 \\
\hline Não & 0 & 0 \\
\hline \multicolumn{3}{|l|}{ Tipos de interrupção } \\
\hline Telefone pessoal tocando & 1 & 0,8 \\
\hline Sobreposição de diferentes tarefas & 4 & 3,2 \\
\hline Interrupção por outro profissional & 116 & 93,5 \\
\hline Telefone institucional tocando & 2 & 1,6 \\
\hline
\end{tabular}

Fonte: Autores (2020).

Em relação à utilização de Equipamentos de Proteção Individual (EPI) pelas enfermeiras, o número de profissionais que utilizaram gorro, máscaras e luvas foram quase unânimes, com exceção de uma que não utilizou luvas de procedimento no momento da diluição de antibióticos.

Tabela 3 - Distribuição percentual, conforme os enfermeiros que utilizaram EPIs no preparo de antibióticos - Fortaleza Ceará, Brasil, 2020.

\begin{tabular}{ccc}
\hline Uso de EPI & $\mathbf{N}$ & $\%$ \\
\hline Utilizam máscaras & 12 & 100 \\
Sim & 0 & 0 \\
Não & & \\
Utilizam luvas & 11 & 91,7 \\
Sim & 1 & 8,3 \\
Não & 12 & 100 \\
Utilizam gorros & 0 & 0 \\
Sim & & \\
Não & &
\end{tabular}

Fonte: Autores (2020).

No que concerne à execução do procedimento, foram observadas a utilização de prescrição pela enfermeira, onde $100 \%$ das observações realizadas evidenciaram a sua não utilização; o rótulo de medicação após a diluição, no qual apenas uma enfermeira não rotulou corretamente; e, por fim, porém bastante significante para a segurança do paciente, a técnica 
empregada na manipulação do antibiótico, onde foi identificado que todas as enfermeiras manipularam de forma incorreta ao menos um dos itens observados, já tornando a técnica como incorreta.

Durante a coleta de dados, foi observado, também, que todos os antibióticos foram preparados com antecedência e armazenados na geladeira em quantidade suficiente para durar o plantão de 12 horas. Assim como, nenhuma enfermeira realizou a desinfecção do frasco-ampolas antes do preparo. Vale destacar ainda que nenhum antibiótico fotossensível foi diluído e observado nesse estudo.

Tabela 4 - Distribuição percentual, conforme a execução do procedimento observado com a utilização de prescrição, rotulação e técnica correta utilizada no preparo de antibióticos - Fortaleza, Ceará, Brasil - 2020.

\begin{tabular}{|c|c|c|}
\hline Procedimentos & $\mathbf{N}$ & $\%$ \\
\hline \multicolumn{3}{|c|}{ Utilização da prescrição médica durante o preparo de medicamentos } \\
\hline Usou prescrição & 0 & 0,0 \\
\hline Não usou prescrição & 12 & 100,0 \\
\hline \multicolumn{3}{|l|}{ Rótulo do medicamento } \\
\hline Rotulou corretamente & 11 & 91,7 \\
\hline Não rotulou corretamente & 1 & 8,3 \\
\hline Não rotulou & 0 & 0,0 \\
\hline \multicolumn{3}{|l|}{ Técnica correta de manipulação } \\
\hline Sim & 0 & 0 \\
\hline Não & 12 & 100 \\
\hline \multicolumn{3}{|l|}{ Técnicas de manipulação observadas } \\
\hline \multicolumn{3}{|l|}{ Lavagem as mãos } \\
\hline Sim & 3 & 25 \\
\hline Não & 9 & 75 \\
\hline \multicolumn{3}{|l|}{ Desinfecção de frasco-ampolas } \\
\hline Sim & 0 & 0 \\
\hline Não & 12 & 0 \\
\hline \multicolumn{3}{|l|}{ Reutilização de materiais (seringas e agulhas) } \\
\hline Sim & 9 & 75 \\
\hline Não & 3 & 25 \\
\hline \multicolumn{3}{|l|}{ Contaminação de material e/ou medicamento } \\
\hline Sim & 8 & 66,7 \\
\hline Não & 4 & 33,3 \\
\hline \multicolumn{3}{|c|}{ Preparo com muita antecedência (acima de 30 min) da administração } \\
\hline Sim & 12 & 100 \\
\hline Não & 0 & 0 \\
\hline Não proteção de medicamentos fotossensíveis & - & - \\
\hline
\end{tabular}

Fonte: Autores (2020).

\section{Discussão}

Os resultados demonstraram que a média de idade foi de 37,83 anos, fase chamada de "Maturidade Profissional" na qual o enfermeiro é assertivo em suas escolhas de emprego, onde o conhecimento, habilidade técnica e qualificação andam atrelados, assumindo sua identidade profissional, almejam o reconhecimento com prosperidade financeira (Machado et al., 2015).

Temos como resultado desta pesquisa que 58,3\% dos profissionais possuem o título de especialista, mestre ou doutor, ou seja, mais da metade das pesquisadas têm pós-graduação concluída. Mesmo após duas décadas da publicação da pesquisa anteriormente citada, apenas uma enfermeira possui o título de mestre e um quantitativo elevado de enfermeiras possui apenas 
o título de graduada $(41,7 \%)$.

Segundo o artigo de Kakehashi et al. (1996), que prevê tendências para o futuro da enfermagem em neonatologia no século XXI, a autora conclui que a aquisição de uma assistência de qualidade e de baixo custo, vem mediante a qualificação profissional do enfermeiro com o título de especialista em neonatologia. Ainda refere que as enfermeiras estadunidenses, com a chegada do novo século, teriam no mínimo a titulação de mestre, mas que essa realidade seria diferente para as enfermeiras brasileiras.

As médias de tempo de formação acadêmica e de experiência profissional neste estudo foram, respectivamente, 12,3 anos e 8,7 anos. E o vínculo empregatício hospitalar mais prevalente foi o de profissionais cooperados (75\%) que acaba desasegurando os direitos trabalhistas reconhecidos em lei, sujeitando o enfermeiro a acumular várias funções, para além do seu exercício profissional, o cenário de atuação torna-se competitivo, ao qual o acúmulo de capital é destaque, nesta lógica neoliberal (Souza et al., 2017).

Em um estudo atual identificou os riscos que a sobrecarga de trabalho na enfermagem destacando-se o acúmulo de funções, a carga horária de trabalho excedente, o não reconhecimento profissional, a não valorização econômica estes repercutem diretamente na segurança do paciente e principalmente, quando são abordados os erros de medicações e as quedas dos pacientes (Pereira et al., 2018).

Assim como as relações de vinculação institucional são importantes, as condições de trabalho também são relevantes para a atuação do enfermeiro assistencial. Dito isso, destacamos que as normas regulamentadoras (NR) funcionam como um guia para licitar as atividades trabalhistas garantindo a segurança do paciente e a saúde do trabalhador no seu local de exercício profissional (Brasil, 2019).

Ambientes hospitalares seguros, permeados por cultura de segurança e boas práticas no manejo de medicamentos, garantem ambientes iluminados, limpos, com controle de pragas, livre de interrupções, com equipamentos e mobiliários que possibilitem controle de ruídos para que a administração de medicamentos ocorra no tempo certo, na dose certa, sem alteração da sua estabilidade física ou química (Feldman et al., 2017).

Portanto, a iluminação tem considerável importância no conforto da paciente e do profissional de saúde. Para além desses fatores, ao se tratar de antibióticos, iluminação acima dos valores limitados pelos fabricantes pode contribuir para a degradação das moléculas do medicamento. A fotólise altera a identidade molecular do fármaco, causando reações de decomposição que alteram a estabilidade e por consequência do prazo de validade da medicação (Brasil, 2013).

A iluminação deve ser projetada de acordo com as necessidades e características dos usuários e do espaço, tarefas visuais e critérios estéticos. Conforme a Agência Nacional de Vigilância Sanitária (ANVISA), uma boa iluminação propicia aumento de produtividade, melhora a qualidade do produto, diminui o desperdício de materiais e reduz a fadiga ocular (Boeck et al., 2019). No presente trabalho, a iluminação foi avaliada em relação à deficiência ou não de luminosidade para o profissional que estava preparando os antibióticos, tendo encontrando, como resultado, a adequação luminosa para todos os enfermeiros. O que contribui, afora a segurança, para o seu conforto.

Além da iluminação, o ruído pode acarretar sérias perturbações funcionais ao organismo influenciando diretamente na produtividade do profissional (Boeck et al., 2019). O ruído aumenta a ocorrência de erros no preparo da medicação, sendo necessário mensurar o seu nível frequentemente, principalmente no local de preparo das medicações, pois exige mais concentração (Pereira et al., 2018).

A NR 15, que trata de atividades e operações insalubres, define ruído contínuo ou intermitente como sendo aquele que não seja causado por impacto. A mesma norma ainda define limites de exposição ao ruído, considerando oito horas o tempo máximo de exposição para níveis de ruído de 85 dB. Já para o Ministério da Saúde, a adequação sensorial auditiva no ambiente neonatal deve estar em torno de 50dB, propiciando uma UN acolhedora (Brasil, 2019). 
Neste estudo, foi possível identificar que, em todas as preparações de medicamentos, o ruído foi classificado como inadequado, aumentando a possibilidade de erros durante o preparo da medicação. Fica evidente que o nível sonoro dentro das unidades neonatais é com frequência elevada, os profissionais discutem casos, solicitam materiais, realizam orientações, movimentam incubadoras, berços térmicos e comuns, o que acabam transformando níveis sonoros saudáveis em ruído, sendo prejudicial aos RNs e aos próprios profissionais (Brasil, 2019).

Para além do ruído, foi percebido que a interrupção no preparo de medicação aconteceu em todos os procedimentos observados, causado por telefone pessoal ou institucional tocando, sobreposição de tarefas diferentes e principalmente, por interrupção por outro profissional. Totalizando 116 interrupções em um total de 12 observações.

É conhecido que quando há interrupções, o erro de medicação é passível de acontecer mediante a quebra da continuidade e concentração do profissional que se encontra manejando o fármaco (Santana et al., 2019). Vale ressaltar que o preparo de medicação é a penúltima barreira antes de a medicação chegar ao paciente, e que para minimizar o erro é fundamental a realização da dupla checagem em todo o processo, assim como, coletes que sinalizem: "não interrompa" (Santana et al., 2019). Estas são estratégias para aperfeiçoar a rotina do enfermeiro nas instituições de saúde.

Contribuindo com conforto do ambiente hospitalar, o espaço utilizado para diluição de medicamentos deve ser ergonômico e planejado para execução do procedimento, preservando a saúde do trabalhador. Organizar o ambiente de preparo de medicamento livre de distrações permite a concentração do profissional (Brasil, 2012). Parte dos enfermeiros observados neste estudo não organizou adequadamente o ambiente de preparo de antibióticos.

A higienização das mãos não é uma opção, é um ato crítico de responsabilidade e honestidade que deve ser um hábito entre os profissionais da saúde, assim como a utilização de equipamentos de proteção individual (EPI). Em uma pesquisa realizada apenas com enfermeiros que teve como objetivo analisar a adesão da equipe de enfermagem à utilização dos equipamentos de proteção individual resultou que $80 \%$ dos enfermeiros utilizaram EPI no preparo de medicação (Sousa et al., 2020).

Neste estudo, todos os profissionais que foram observados durante o preparo de antibióticos dispunham de local adequado para higiene das mãos. Porém, nem todos higienizaram as mãos (25\%) e apenas um foi observada não utilizou luvas de procedimento para execução da diluição de medicamentos.

Afora a higiene das mãos, a limpeza de superfícies e bancadas são fundamentais para o preparo de medicamentos sem contaminação. As bancadas, segundo a classificação de áreas em serviços de saúde, são consideradas áreas não-críticas, onde não entram em contato com pacientes e secreções humanas com alta ou baixa transmissibilidade de doenças infecciosas (Brasil, 2012).

O manual de Segurança do Paciente em Serviços de Saúde: limpeza e desinfecção de superfícies orientam que as bancadas e superfícies sejam limpas com água e sabão ou detergentes, enxugadas e secadas; ou desinfetadas com fricção de álcool a 70\% ou ainda, com a utilização de outro desinfetante definido pelo Serviço de Controle de Infecção Hospitalar (Brasil, 2013).

Considerando o exposto no referido manual, a limpeza da superfície de preparo neste estudo foi realizada inadequadamente $(58,3 \%)$, ou não foram higienizadas e/ou foi colocado um pano não estéril em cima da bancada. O manual ainda afirma que a desinfecção realizada com álcool é a mais indicada, devido a sua fácil aplicação e ação imediata eliminando bactérias, vírus, fungos e bacilo da tuberculose, agindo através da desnaturação das proteínas da parece celular, com a desvantagem de não ser esporicida (ANVISA, 2012).

Corroborando com a biossegurança, os frascos multidoses proporcionam perfurações sucessivas. Por isso, necessitam de higienização com álcool a 70\% antes da aspiração do conteúdo e não pode haver reutilização de descartáveis, seringas e agulhas devido ao grau de contaminação que é provável fonte de infecção nosocomial. Um estudo iraniano sobre a 
contaminação em frascos multidoses, evidenciou que houve predominância de bactérias gram-positivas (88,9\%) com maior frequência para Staphylococcus epidermidis $(44,4 \%)$ e menor para Actinomyces viscosus $(2,8 \%)$ nos frascos analisados (Motamedifar \& Askarian, 2009).

Segundo o Protocolo de segurança na prescrição, uso e administração de medicamentos, a utilização do sistema coletivo de distribuição de medicamentos que ocorre com a formação de subestoques dentro da unidade sob responsabilidade da equipe de enfermagem, deve ser abolido nas instituições de saúde, pois ressalta-se que além de ser inseguro, é um provável gerador de erro na assistência (Brasil, 2012).

Vale ressaltar, ainda, que o mesmo protocolo orienta que as medicações sejam distribuídas por dose unitária, reduzindo a manipulação antes da administração e que os medicamentos sejam dispensados em horários específicos, utilizando o aprazamento para aplicar horários padrões institucionalizados, pois medicações em intervalos não padronizados estão propensas ao esquecimento, causando oscilação da dose do medicamento no organismo (Oliveira et al., 1996).

Neste estudo, todas as prescrições de antibióticos são transcritas para uma folha padronizada da instituição, contendo: nome da medicação, frequência de aprazamento, apresentação, via de administração e duração. Em um artigo publicado em 1986 sobre transcrição da prescrição médica x assistência de enfermagem conclui que o tempo gasto para transcrição dos medicamentos é elevado, ocorrem frequentes interrupções que favorecem o erro por descontinuidade no processo e sugere que haja maneiras de aperfeiçoar e mudar o sistema de transcrição de prescrição (Oliveira et al., 1996).

Trinta e quatro anos após esta publicação, o procedimento de transcrição de medicamentos ainda existe. Sistemas que usam a Tecnologia da informação (TI) aperfeiçoam a assistência em saúde, melhorando o acesso à informação com organização dos dados. Um estudo sobre prevenção dos erros de medicação através de um sistema de TI explana que acontece 12\% de erro no momento da transcrição com competência de interceptar 33\% dos erros (Agrawal et al., 2009).

A técnica de manipulação é fundamental para o cuidado seguro, obtivemos que nenhuma manipulação de medicação foi feita com a técnica correta, conforme exposto na tabela 4. Destaca-se aqui que a não desinfecção dos fracos-ampolas e o preparo da medicação com muita antecedência (acima de 30 minutos) da administração foram unânimes.

De acordo com o COREN de São Paulo que aborda o uso seguro de medicamentos no "Guia para preparo, administração e monitoramento", refere que é necessário higienizar as mãos antes e depois do preparo de medicação, bem como a limpeza do diafragma do frasco-ampola ou frasco de multidoses com álcool a 70\% antes de perfura-lo; manipular materiais de preparo cumprindo a técnica segura de manipulação para evitar contaminação; e, por fim, os antimicrobianos devem ser preparados e administrados em prazos específicos, visto que são medicamentos classificados como programados de tempo crítico, onde a administração fora do intervalo de 30 minutos, antes ou depois do horário prescrito, pode causar danos ao paciente (Feldman et al., 2017; Brasil, 2012).

Além do exposto, para a identificação do medicamento, administrado imediatamente após o preparo, é necessário que a etiqueta contenha o nome do paciente, o nome do medicamento, a dose e a via a ser administrada (Feldman et al., 2017). Entretanto, neste estudo, todas as medicações foram armazenadas em geladeira após reconstituição e rediluição, quase todas (91,7\%) contiveram as informações: data e horário do preparo, concentração, via de administração, autor do preparo e validade da medicação, exceto uma que não tinha a validade do medicamento.

Por fim, revisar minuciosamente o saber-fazer em neonatologia é de enorme valia ao se tratar de RN população delicada, onde qualquer manejo inadequado, não conformidades, no cuidado prestado pode ocasionar atraso no crescimento e desenvolvimento, e até mesmo levar a morte. Humanizar a assistência, é ver o RN integralmente, é abrigá-lo em todos os âmbitos, bio-psico-socio-cultural, desde o nascimento.

O tempo em que a pesquisa foi realizada, apenas um mês e a quantidade de vezes em que foi observado o preparo de medicação, no caso, apenas uma por profissional, são limitações relevantes do estudo. Estas podem ter restringido um maior 
acúmulo de informações a respeito da temática e até mesmo notar mudanças de condutas por parte dos profissionais, a partir das informações passadas em caso de erros no preparo de antibióticos. Embora a pesquisa tenha cumprido com o seu objetivo.

\section{Conclusão}

Pela observação dos aspectos aqui estudados, os principais achados com relação ao ambiente estão relacionados à iluminação; ao uso de EPIs, as máscaras e gorros; e quanto a aplicação da semiotécnica, não houve nenhuma conformidade. Salienta-se que as interrupções ao enfermeiro no preparo de medicação é uma falha grave e corriqueira que compromete a segurança do paciente.

As não conformidades se sobressaem e nos levam a refletir sobre a atuação profissional do enfermeiro com vínculo empregatício frágil e no poder da gestão de interferir positivamente na promoção de um ambiente seguro, desencadeado por uma cultura de segurança institucionalizada.

Sugere-se que novos estudos possam ser realizados com o intuído de ser melhor apresentado e poder contribuir através do estimulo a implementação de políticas públicas que venham transformar a forma do cuidado, e assim abrir espaço para atualizar a equipe de enfermagem na aplicação de boas práticas no preparo de medicamentos. Recursos físicos e humanos, qualificados, é a díade que permeia as boas práticas no manejo adequado de antibióticos na unidade neonatal, resultando no cuidado seguro ao recém-nascido.

\section{Referências}

Agrawal, A. (2009). Medication errors: prevention using information technology systems. British Journal of Clinical Pharmacology; 67(6): 681-686.

Boeck, K. H., Hiittl, N., Cembranel, P., \& Taschetto, L. (2019). A segurança do paciente devido os riscos da sobrecarga de trabalho dos enfermeiros. Revista de Administração Hospitalar e Inovação em Saúde; 16(3):15-27. https://doi.org/10.21450/rahis.v16i3.5993

Cassiani, S. H. B., Teixeira, T. C. A., Opitz, S. P., Silva, A. E. B. C., Gimenes, D. R. E., \& Fakih, F. T. (2006). Identificação e análise dos erros de medicação em seis hospitais brasileiros. Ribeirão Preto: EERP.

Embree, J. E., \& Alfattoh, N. I. Infecção no recém-nascido. In: Macdonald MG, Seshia MK. (2018). Avery neonatologia, fisiopatologia e tratamento do recém-nascido. ( $7^{\mathrm{a}}$. ed.): Guanabara Koogan,. 980 - 1036.

Feldman, L. B., Harada, M. J. C. S., Gabriel, C. S., Schmidt, E. A., \& Cadah, L. (2017). Uso seguro de medicamentos: guia de preparo, administração, monitoramento: Coren-SP.

Guzzo, G. M., Magalhães, A. M. M., Moura, G. M. S. S., \& Wegner, W. (2018). Segurança Da Terapia Medicamentosa Em Neonatologia: Olhar Da Enfermagem Na Perspectiva Do Pensamento Ecológico Restaurativo. Texto Contexto Enferm. [Internet], 27(3): e4500016. https://doi.org/10.1590/0104070720180004500016 .

Kakehashi, T. Y. (1996). A situação atual da Enfermagem Neonatológica e suas tendências para o futuro. Acta Paul Enferm., 9(special issue), 17-22.

Machado, M. H., Aguiar Filho, W., Lacerda, W. F., Oliveira, E., Lemos, W., Wermelinger, M., et al. (2015). Caracteristicas Gerais Da Enfermagem: O Perfil Sócio Demográfico. Enfermagem em Foco 7(ESP):09-14.

Ministério da Saúde. Agência Nacional de Vigilância Sanitária. (2012). Protocolo de segurança na prescrição, uso e administração de medicamentos. Diário Oficial da União, Brasília https://www20.anvisa.gov.br/segurancadopaciente/index.php/publicacoes/item/seguranca-na-prescricao-uso-e-administracao-demedicamentos

Ministério da Saúde. Agência Nacional de Vigilância Sanitária. (2013). Medidas de prevenção de infecção relacionada à assistência à saúde. Diário Oficial da União, Brasília. https://www20.anvisa.gov.br/segurancadopaciente/images/documentos/livros/Livro4MedidasPrevencaoIRASaude.pdf

Ministério da Saúde. Secretaria de Atenção à Saúde. (2018). Atenção humanizada ao recém-nascido: método Canguru: manual técnico. Diário Oficial da

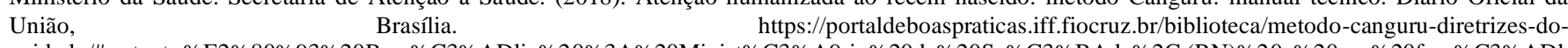
cuidado/\#: :text=\%E2\%80\%93\%20Bras\%C3\%ADlia\%20\%3A\%20Minist\%C3\%A9rio\%20da\%20Sa\%C3\%BAde\%2C,(RN)\%20e\%20sua\%20fam\%C3\%AD1 ia

Ministério do Trabalho e Emprego. Gabinete do Ministro. (2019). Normas Regulamentadoras de Segurança e Medicina do Trabalho. NR 15 Atividades e Operações Insalubres. Diário Oficial da União, Brasília. http://www.mte.gov.br

Motamedifar, M., \& Askarian, M. (2009). The prevalence of multidose vial contamination by aerobic bacteria in a major teaching hospital, Shiraz, Iran, 2006. Am J Infect Control; 37(9):773-7. 10.1016/j.ajic.2008.12.009 
Research, Society and Development, v. 10, n. 5, e34610515084, 2021

(CC BY 4.0) | ISSN 2525-3409 | DOI: http://dx.doi.org/10.33448/rsd-v10i5.15084

National Coordinating Council For Medication (2015). Error Reporting And Prevention [homepage na internet]. Definition medication erros. https://www.nccmerp.org/about-medication-errors.

Oliveira, A. C., Pereira, M. S., Pereira, M. P. M., \& Faustino, L. H. (1986). Transcrição da prescrição médica x assistência de enfermagem. Ver. Bras. Enf, $39(2 / 3): 12-15$.

Organização das Nações Unidas (ONU). Erros no uso de medicações custam US\$42 bi por ano no mundo, estima ONU; 2017. https://nacoesunidas.org/errosno-uso-de-medicacoes-custam-us42-bi-por-ano-no-mundoestima-onu /.

Pereira, F. G. F., Aquino, G. A., Melo, G. A. A., Praxedes, C. O., \& Caetano, J. Á. (2016). Conformidades e não conformidades no preparo e administração de antimicrobianos. Cogitare Enferm, 21(esp):1-9.

Pereira, F. G. F., Caetano, J. Á., Ataíde, M. B. C., Silva, R. L., Néri, E. D. R., \& Carvalho, G. C. N. (2018). Environmental variables and errors in the preparation and administration of medicines. Rev Bras Enferm [Internet];71(3):1109-17. http://dx.doi.org/10.1590/0034-7167-2016-0041

Popescu, A., Currey, J., \& Botti, M. (2011). Multifactorial influences on and deviations from medication administration safety and quality in the acute medical/ surgical context. Worldviews Evid Based Nurse, 1(8):15-24.

Santana, B. S., Rodrigues, B. S, Stivel, M. M., Rehem, T. C. M. S. B., Lima, L. R., \& Volpe, C. R. G. (2019). Interrupções no trabalho da enfermagem como fator de risco para erros de medicação. Av. Enferm; 37(1): 56-64. https://doi.org/10.15446/av.enferm.v37n1.71178

Souza, N. V. D. O., Pires, A. S., Gonçalves, F. G. A., Tavares, K. F. A., Baptista, A. T. P., \& Bastos, T. M. G. (2017). Formação em enfermagem e o mundo do trabalho: percepções de egressos de enfermagem. Aquichan., 17(2):204-216. 10.5294/aqui.2017.17.2.9.

Sousa, F. C. A., Oliveira, M. L. V., Siqueira, H. D. S., Siqueira, F. F. F. S., Silva, W. C., \& Rodrigues, L. A. S. (2020). Adesão ao uso dos equipamentos de proteção individual pela equipe de enfermagem no ambiente hospitalar. Res., Soc. Dev., 9(1):e59911607.

WHO. World Health Organization. Medication Without Harm - Global Patient Safety Challenge on Medication Safety. Geneva: World Health Organization; 2017. http://www.gims-foundation.org/wp-content/uploads/2017/05/WHO-Brochure-GPSC_Medication-Without-Harm-2017.pdf 